Cahiers d'études italiennes

\title{
La violence du Pouvoir : le regard de Pier Paolo Pasolini
}

Flaviano Pisanelli

\section{(2) OpenEdition}

\section{Journals}

Édition électronique

URL : http://journals.openedition.org/cei/277

DOI : $10.4000 /$ cei. 277

ISSN : 2260-779X

Éditeur

UGA Éditions/Université Grenoble Alpes

Édition imprimée

Date de publication : 15 juin 2005

Pagination : 89-114

ISBN : 978-2-84310-066-6

ISSN : $1770-9571$

\section{Référence électronique}

Flaviano Pisanelli, «La violence du Pouvoir : le regard de Pier Paolo Pasolini », Cahiers d'études italiennes [En ligne], 3 | 2005, mis en ligne le 15 décembre 2006, consulté le 27 mars 2021. URL : http:// journals.openedition.org/cei/277 ; DOI : https://doi.org/10.4000/cei.277 


\title{
LA VIOLENCE DU POUVOIR \\ Le REGARD de Pier Paolo Pasolini
}

\author{
Flaviano Pisanelli \\ Université d'Avignon
}

\begin{abstract}
Scrivo « Potere » con la P maiuscola [...] solo perché sinceramente non so in cosa consista questo nuovo Potere e chi lo rappresenti. So semplicemente che c'è.

Non lo riconosco più nel Vaticano, né nei Potenti Democristiani, né nelle Forze Armate. Non lo riconosco Più neanche nella grande industria $[\ldots]:$ a me, almeno, essa appare piuttosto come un tutto (industrializzazione totale), e, per di più, come tutto non italiano (transnazionale) ${ }^{1}$
\end{abstract}

Entre 1968 et 1978, l'Italie vit une décennie très difficile aux niveaux social, culturel et politique : l'exigence d'un renouvellement de la société devient de plus en plus évidente, les mouvements ouvriers découvrent de plus en plus leur rôle politique et leur identité spécifique, les étudiants rêvent d'une société plus moderne et ouverte aux valeurs laïques. En même temps, n'arrivant pas à faire face aux différentes situations qui se profilent, la classe politique laisse un vide de pouvoir dont certaines formations extrémistes nouvellement créées profiteront plus tard, tout en mettant en œuvre une stratégie de la terreur fondée sur la violence et le désordre.

Les intellectuels se taisent et, sans proposer un quelconque modèle culturel capable d'interpréter les événements qui se succèdent, ni comprendre cette société qui évolue et met pour la première fois en crise le

1. Pier Paolo Pasolini, Scritti corsari, Milan, Garzanti, 1990, p. 45-46. "J'écris "Pouvoir" avec un P majuscule [...] parce que, sincèrement, je ne sais pas en quoi consiste ce nouveau Pouvoir et qui le représente. Je sais simplement qu'il existe. Je ne le reconnais plus dans le Vatican, dans les Grands démocrateschrétiens, ni dans les forces armées. Je ne le reconnais pas davantage dans la grande industrie [...] ; en ce qui me concerne, elle m'apparaît plutôt comme un tout (industrialisation totale), et même comme un tout non italien (transnational) ». Pier Paolo Pasolini, Écrits corsaires, trad. fr. par Philippe Guilhon, Paris, Flammarion, 1976. 
système politique et social, ils passent leur temps à débattre de telle ou telle idéologie de manière assez stérile. La politique menée par les catholiques ne répond plus aux attentes de la majorité des Italiens et, en même temps, le Parti communiste déçoit énormément les ouvriers et les classes sociales les plus démunies. Il aurait fallu à ce moment-là une réflexion culturelle généralisée, une remise en question des différentes idéologies, au-delà des notions de conservatisme ou de révolution autour desquelles allait s'entamer un débat sans issue. Aucune force politique n'était capable de faire face à l'impuissance de la Démocratie chrétienne, qui résistait toutefois en tant que premier parti du pays.

Dans cette même période, on assiste à la naissance de mouvements d'opposition sociale qui, tout d'abord, s'organisent au sein des usines du nord de l'Italie et dans certaines universités (Trente et Turin), pour se répandre ensuite dans tout le pays. De nombreux mouvements anarchistes et révolutionnaires se constituent alors pour lutter à la fois contre une Démocratie chrétienne anachronique et contre un Parti communiste qui, aux yeux des militants de la gauche la plus radicale, représente une institution dépourvue de toute action sociale. Dans ce contexte de désordre généralisé poussant à la mobilisation des masses, Pasolini, comme un petit nombre d'autres intellectuels, a toujours tenté de faire entendre sa voix, tout en proposant une figure d'intellectuel libre et difficile à classer dans une catégorie idéologique et politique précise.

Il consacre une grande partie de sa dernière production littéraire à la représentation et à la connaissance des causes, des effets, et surtout des raisons de la violence sociale, politique et culturelle qui éclate en Italie à partir de 1969. À travers son écriture, Pasolini assume le rôle de « leader d'opinion » engagé, faisant de la littérature un lieu de réflexion et d'évaluation pour interpréter enfin librement l'évolution d'une société et d'une culture qui contribuent à réaliser, depuis la fin des années 50, un projet voué à la "standardisation " culturelle et sociale, à la consommation et à l'idée d'un développement sans la moindre référence au progrès.

\section{La violence du pouvoir : l'intellectuel face au nouveau pouvoir}

Plus que d'un intellettuale integrale (si l'on se réfere à la définition donnée par Gramsci dans les Quaderni dal carcere, qui décrit le profil de l'intellectuel proche des exigences de la classe ouvrière, opposé à l'intellectuel traditionnel, représentant plutôt le point de vue de la classe dominante) on 
préfere parler de Pasolini comme d'un intellectuel disorganico. Même s'il idéalise le sous-prolétariat urbain et la culture paysanne, qui constituent à ses yeux l'alternative à la société post-moderne de consommation, les seuls symboles d'innocence et de pureté, il ne cesse en effet d'aborder les sujets concernant la culture, l'art, la politique, la sémiologie, ou encore les techniques d'information, d'une manière tout à fait "disorganica ». Autrement dit, il demande toujours au lecteur ou à l'interlocuteur d'être traduit et non pas d'être critiqué scientifiquement ou politiquement. Il emploie des langages différents, appartenant à des domaines parfois autres que le domaine littéraire, sans jamais cesser d'être un poète. Il ne parle jamais en tant que spécialiste d'une discipline ; bien au contraire, il emploie métaphoriquement les discours scientifiques, philologiques, linguistiques et politiques. Pendant les dernières années de sa vie, Pasolini est intervenu de manière polémique sur plusieurs sujets d'actualité : or, il n'a jamais été interprété ni traduit comme il le souhaitait; en revanche, il a été lu, sur le thème dont il parlait (loi sur le divorce, loi sur l'avortement, etc.). En raison de son langage polémique, une mauvaise lecture a souvent été faite de son œuvre, surtout de ses écrits posthumes ${ }^{2}$.

L'attention que Pasolini porte aux problèmes politiques et sociaux et à la violence qui se manifeste en Italie pendant les années 60 et 70 est évidente, surtout dans les Écrits corsaires. Grâce à ces articles, l'auteur dénonce le vide laissé par une classe politique et une Église désormais incapables de proposer une culture, quelle qu'elle soit, de comprendre la majorité de la population, de saisir la portée de la révolution conformiste invisible, qui ne s'oppose au pouvoir ancien que pour en proposer un autre encore plus écrasant : celui qui impose la disparition de toute culture et de toute tradition au nom d'une consommation et d'un développement ne prévoyant aucune forme de progrès. En tant que communiste, Pasolini, comme il le déclare lui-même en 1963, puis en 1975, met également en évidence les limites d'une gauche italienne ne s'opposant au pouvoir qu'à travers un système moral et politique qui n'apporte à l'époque rien d'actuel ni de nouveau. Face à cette situation, les intellectuels ouvraient souvent des débats tout en restant prisonniers de telle ou telle idéologie, sans s'apercevoir que l'Italie n'avait que besoin de témoins pour que son actualité devienne histoire. La société italienne était en demande de véritables

2. De cette dernière production on tiendra compte surtout du recueil poétique Trasumanar e organizzar (Pier Paolo Pasolini, Trasumanar e organizzar, Milan, Garzanti, 1971) et de deux des ouvrages publiés après sa mort : Écrits corsaires (Pier Paolo Pasolini, Scritti corsari, Milan, Garzanti, [1975], 1990) et Pétrole (Pier Paolo Pasolini, Petrolio, Turin, Einaudi, 1992), roman inachevé dont les notes provisoires ont été publiées sous la direction du philologue Aurelio Roncaglia. 
poètes pour " agir " au-delà du simple discours, pour balayer les idées reçues et passivement acceptées tout au long des trente années de vie républicaine, afin de pouvoir répondre à la "standardisation " culturelle qui, dans les années 80 , s'affichera et s'exprimera dans toute sa violence.

Le manque d'une véritable référence, d'une auctoritas capable de créer une confrontation totale entre les différentes cultures du pays, et la nécessité de combler un vide politique et culturel, ont déclenché une vague de violence en quelque sorte autorisée par une classe dirigeante, qui n'avait qu'à suivre cette voie pour imposer un modèle social répondant aux exigences de la logique néocapitaliste. Il s'agissait donc d'une violence, parfois armée, utilisée par un pouvoir agonisant qui a su mêler, à son profit, des principes idéologiques et culturels diamétralement opposés : fascisme et antifascisme, culture de droite et de gauche, progrès et autoritarisme, révolution et conservatisme. Face à une Église impuissante, à une Démocratie chrétienne en déclin et à un groupe d'intellectuels débattant au nom d'idéologies désormais putrescentes, face à la disparition de l'individu et au rêve toujours plus puissant de parvenir à une richesse purement économique, l'absence de pouvoir a été comblée par une bourgeoisie sans scrupules, néocapitaliste, et apparemment tolérante à l'égard des minorités. Elle a su imposer un style de vie commun sous le signe de l'hédonisme au détriment de toute différence culturelle ${ }^{3}$.

Dans Écrits corsaires ${ }^{4}$, Pasolini illustre et attaque très violemment cette société déshumanisée et déshumanisante, ce modèle de développement qui transforme les classes sociales vivant à l'écart, dans les faubourgs des grandes villes et les villages ruraux, et qui échappaient encore aux logiques de la consommation, aux mythes de la fausse tolérance, à l'embourgeoisement, à ce que Pasolini définit comme le «nouveau fascisme».

3. Pasolini soutient à plusieurs reprises que l'Église elle-même était devenue inutile au pouvoir bourgeois et capitaliste qui fait passer son message par les moyens de communication de masse : les mêmes qui, auparavant, avaient été au service de la culture catholique et démocrate-chrétienne. À la suite de la "standardisation » culturelle en cours, du changement et de la disparition de la culture paysanne et ouvrière, l'Église a perdu une partie importante de ses " adeptes ». Pour éviter une crise irréversible, l'institution catholique n'avait qu'à passer à l'opposition pour combattre le nouveau pouvoir de la consommation, complètement irréligieux, totalitaire, violent, faussement tolérant, corrupteur et dégradant, et qui s'impose comme seule alternative sociale. À propos de cette " révolution " des mœurs et du rapport entre l'Église et la nouvelle société de consommation cf. en particulier les articles suivants : "Le petit discours historique de Castelgandolfo » (p. 117-123), "Nouvelles perspectives historiques : l'Église est inutile au Pouvoir» (p. 124-130), "L'ignorance vaticane comme paradigme de l'ignorance de la bourgeoisie italienne " (p. 138-142), "Enrichissement de l'essai sur la révolution anthropologique en Italie " (p. 89-100), in Ecrits corsaires, trad. fr. par Philippe Guilhon, op. cit.

4. Pier Paolo Pasolini, Ecrits corsaires, op. cit. 
L'ouvrage présente donc un ensemble d'écrits dont l'organisation, voulue par l'auteur, révèle une structure très calculée et très rigoureuse. Pourtant, dans la Note introductive, il invite le lecteur à reconstituer la structure du livre, qui ne se présente que comme un ensemble de notes, de fragments, d'hypothèses divergentes ${ }^{5}$. Le discours qu'il propose ne s'appuie sur aucune idéologie préexistante : il exprime une dissidence viscérale, un vécu inclassable. Toutes les problématiques évoquées (notamment la situation de la bourgeoisie italienne, la renonciation de l'Église à son rôle de guide spirituel, le vide du pouvoir qui efface toute différence entre droite et gauche, l'hédonisme sexuel qui s'exprime à travers la loi sur l'avortement), se traduisent en images dont la caractéristique est d'être des visions nées d'un excès de rage et, en même temps, de dérision.

Dissimulant ce vide de pouvoir dont on a mesuré l'importance, la violence éclate en Italie surtout autour des mouvements ouvriers, acteurs de l'" automne chaud» de 1968, et autour des universités, à plusieurs reprises occupées par les manifestants. La position de Pasolini est très complexe. Lors des premiers affrontements entre la police et les étudiants, il n’hésite pas à soutenir les jeunes policiers, notamment dans un poème publié par $L^{\prime} E s p r e s s{ }^{6}$. Ce poème a permis à ses détracteurs de le traiter de conservateur, sans comprendre que son analyse dépassait la notion même d'idéologie, tout en mettant en crise le marxisme, très mal interprété, y compris par le Parti communiste de l'époque. Pasolini considérait que le gigantesque phénomène d'acculturation en cours (notamment le grand nombre d'étudiants présents dans les universités) ne représentait pas le libre accès à la culture ni le moyen d'un rachat social de la part du sous-prolétariat, mais, au contraire, la cause de la complète disparition de cette classe sociale. Il s'agissait du véritable " fascisme d'aujourd'hui ", d'une occasion

5. Le lecteur se retrouve face à un livre qui se compose de deux types d'écrits : les premiers, publiés dans le Corriere della Sera, présentent le débat officiel concernant les sujets d'actualité les plus importants ; viennent ensuite les écrits littéraires, qui devraient soutenir et argumenter de manière critique les premiers. La réalité n'est plus conçue comme un ensemble partagé entre l'idée du bien et du mal. Il n'y a plus le conformiste capitaliste et celui qui s'oppose à cette réalité, croyant encore à l'efficacité de la lutte des classes. À ce propos, Marco Antonio Bazzocchi écrit : "Si può ipotizzare che gli scritti corsari nascano da un atteggiamento simile. Perdono valore se li leggiamo come l'ennesimo tentativo di Pasolini di opporre passato e presente, mondo contadino e mondo capitalista, passione e storia. Nuovo, e irripetibile, è il modo in cui Pasolini riesce a dare evidenza fisica a quello che dice, a creare immagini forti con cui sintetizzare i concetti, dimostrando di avere ancora un rapporto privilegiato con quella realtà che un atteggiamento borghese non vede neanche più ". In Marco Antonio Bazzocchi, Pier Paolo Pasolini, Milan, Bruno Mondadori, 1998, p. 174-175.

6. "Adesso i giornalisti di tutto il mondo / (compresi quelli delle televisioni) / vi leccano (come ancora si dice nel linguaggio / goliardico) il culo. Io no, cari. / Avete facce di figli di papà / [...] / Siete pavidi, incerti, disperati / [...] / Quando ieri a Valle Giulia avete fatto a botte / coi poliziotti, / io simpatizzavo coi poliziotti. / Perché i poliziotti sono figli di poveri, / vengono da subtopaie, contadine o urbane che siano ». L'Espresso, XIV, n. 24, 16 juin 1968. 
pour s'exposer aux lazzis ou à la hargne de ceux qui ne confiaient le sens de leur vie qu'à une fausse idée de bien-être et de culture. Dans sa préface au volume É crits corsaires ${ }^{7}$, Philippe Gavi explique bien la position inconfortable de Pasolini face aux cultures de droite et de gauche, montrant que, d'un côté, on lui reprochait de regretter une sorte d'âge d'or pré-catholique et que, de l'autre, on voyait en lui le véritable idéologue de la réaction ${ }^{8}$. Cependant, tout au long de sa vie, Pasolini affirmera que la libération et la désobéissance n'ont de sens que si elles s'insurgent furieusement contre ce que la société, par l'intermédiaire de ses instances de pouvoir, exige et impose.

On peut sans doute dire que la violence que le pouvoir feignait de combattre, et le désordre social auquel il fallait s'opposer, constituaient les deux éléments du projet mis en place par ce même pouvoir (qui comprenait non seulement la classe politique dirigeante, l'Église et les autres institutions, mais aussi la grande machine du système de consommation). Pour Pasolini, il n'était plus temps de parler d'idéologies ni de partis politiques, il fallait remettre en cause les principes culturels dont l'Europe occidentale s'était nourrie depuis la fin de la Seconde Guerre mondiale. Plus vite que les autres, Pasolini avait compris qu'on n'était pas devant un problème politique ou social, mais qu'il fallait aborder la question d'un point de vue existentiel et culturel. Écrits corsaires, qui peut être considéré comme un « roman » de la violence du pouvoir, met surtout en avant le déclin et la fin de la jeunesse : enfermée dans son isolement, elle ne sait qu'exprimer - physiquement et moralement - son anxiété devant les modèles de consommation imposés par une société qui a complètement oublié la tradition et l'identité de classe. La véritable violence exercée par ce type de non-pouvoir reste, malgré tout, celle qui a su refuser à jamais aux jeunes la possibilité de vivre leur jeunesse, introduisant l'anxiété de mort dans l'espoir de vivre ${ }^{9}$. Les jeunes ont trahi leur jeunesse au nom d'une fausse liberté, d'une révolte qui était en fait une restauration et, par conséquent, ils ont pris la place de leurs pères : ils n'ont donc pas compris qu'ils avaient tout perdu et servi un non-pouvoir qui leur avait volé leur appartenance à l'histoire.

7. Pier Paolo Pasolini, Écrits corsaires, trad. fr. par Philippe Guilhon, op. cit.

8. Philippe Gavi, Préface à Écrits corsaires, op. cit., p. 11-22.

9. "Io mi guardo indietro, e piango / i paesi poveri, le nuvole e il frumento ; / la casa scura, il fumo, le biciclette, gli aeroplani / che passano come tuoni : e i bambini li guardano ; / il modo di ridere che viene dal cuore ; / gli occhi che guardandosi intorno ardono / di curiosità senza vergogna, di rispetto / senza paura. Piango un mondo morto / Ma non son morto io che lo piango. / Se vogliamo andare avanti, bisogna che piangiamo / il tempo che non può più tornare, che diciamo di no / a questa realtà che ci ha chiusi / nella sua prigione... [...] ". Significato del rimpianto, in Pier Paolo Pasolini, La nuova gioventù, Turin, Einaudi, 1975. 
Pour ces raisons, Pasolini ne pouvait que reprocher aux jeunes et aux étudiants révoltés d'être de simples outils du pouvoir, qu'il utilisait pour réaliser son projet de génocide culturel.

\section{La violence du pouvoir comme génocide culturel}

Le génocide: c'est le titre d'un article publié le 27 septembre 1974 dans Rinascita, et qui fait partie des Écrits corsaires. Il s'agit d'un discours prononcé par Pasolini à l'occasion de la "Festa dell'Unità " qui eut lieu à Milan, au cours de l'été 1974 . On est en pleine époque terroriste : l'Italie avait vécu le massacre de la piazza Fontana à Milan, les attentats de Rome et de Bologne, et une autre vague de violence se préparait avec l'action de groupes armés, comme les Brigades Rouges, qui agiront surtout pendant les années $\mathrm{du}$ "Compromis historique » (notamment avec l'assassinat d'Aldo Moro).

Cependant, quand Pasolini parle de génocide, il affirme que ce phénomène s'exprime de manière plutôt pacifique, sans un pesant recours à la violence. Expliquant la nature de ce phénomène, il fait référence au génocide perpétré par la culture bourgeoise à l'encontre des classes dominées, le sous-prolétariat urbain et certaines populations paysannes. À l'époque du Manifeste de Karl Marx, si cette substitution de valeurs passait par l'usage explicite de la violence (au moyen de la conquête coloniale et de l'imposition par la force), de notre temps le phénomène se produit à travers un processus beaucoup plus pervers. De larges strates de la société, restées longtemps à l'écart de l'histoire (notamment le sous-prolétariat urbain et les paysans du centre et du sud d'Italie), subissent au cours des années 60 et 70 la même agression de la part de la classe sociale dominante - la bourgeoisie - qui impose, depuis la révolution industrielle, sa culture. Cette bourgeoisie a voulu assimiler les classes subalternes à son mode de vie en leur imposant de nouvelles valeurs, de nouveaux besoins à satisfaire, des modèles sociolinguistiques absolument " autres " qui, toutefois, répondent mieux à la logique d'une société de consommation dont elle est l'expression.

Pour expliquer cette situation paradoxale, il faut quitter le langage et l'argumentaire politique des Écrits corsaires, et se pencher sur un autre ouvrage que Pasolini écrivait à l'époque, et qui est resté inachevé en raison de sa mort prématurée : Pétrole.

Lors des premiers mois de rédaction de l'ouvrage, Pasolini écrit à Moravia : 
E' un romanzo, ma non è scritto come sono scritti i romanzi veri : la sua lingua è quella che si adopera per la saggistica, per certi articoli giornalistici, per le recensioni, per le lettere private e anche per la poesia ${ }^{10}$.

Pétrole, qui était considéré par l'auteur lui-même comme la somme de ses expériences et de ses souvenirs, va au-delà de l'idée traditionnelle d'œuvre littéraire : c'est un hypertexte qui mêle différents types d'écriture aux genres littéraires les plus variés ${ }^{11}$. Pasolini n'écrit pas une histoire, ne fonde pas l'intrigue sur des personnages précis : l'actualité historique pénètre dans la fiction littéraire, le langage journalistique croise les argumentations propres à un essai critique, et la poésie qui se cache derrière les passages oniriques nourrit les réflexions issues de l'observation d'une réalité de plus en plus difficile à cerner. Pétrole consacre irrémédiablement l'impossibilité, pour l'écrivain, de représenter le monde extérieur et, en même temps, la tentative de cet intellectuel disorganico de mettre en œuvre une écriture adhérant à la réalité et dénonçant la disparition de l'homme dans une société gérée par un pouvoir sourd et muet, poussant à l'aphasie et à la perte de toute différence culturelle. Ce " roman » se fonde sur une parole qui agit et analyse, tout en réalisant une synthèse de poétiques ayant la force - surtout grâce à la puissance de l'allégorie - de retrouver la possibilité de dire et de combler le silence créé par l'absence d'un langage apte à s'opposer au nivellement social en cours.

Le titre, "Pétrole ", explique déjà l'origine et la " matière première » du "roman " : c'est une analyse profonde de la société de consommation, des logiques du capitalisme et de la dégradation de l'homme devenu une pure marchandise ${ }^{12}$. Le pétrole devient le symbole par excellence de l'époque

10. Petrolio, op. cit., p. 544.

11. À propos de la structure de Pétrole, on parle souvent d'un roman impossible, d'un méta-roman philologique, d'un long poème en prose dirigé par une voix, en même temps narrative et portée à la réflexion. La fonction méta-littéraire due à l'insertion à l'intérieur de l'ouvrage de nouvelles, l'emploi permanent de l'allégorie comme expression définitive du mystère, la qualité du conte toujours placé entre la sphère réaliste et la sphère onirique, la référence à l'écriture de Dostoïevski et au caractère ambigu de l'œuvre de Pound, ainsi que la notion de comique et le recours à un langage cinématographique, font de Pétrole un lieu de rencontre de genres et de modèles littéraires très différents. Pasolini crée une expérimentation stylistique et linguistique intense dans le but de contrevenir à toute idée de Forme et de convention littéraire. "Il disordine, la polivocità, l'enigmaticità si profilano dunque come dati strutturali del testo, non soltanto come condizioni contingenti derivate dall'incompiutezza, ma come valori che dovranno organizzare i significati narrativi, sia sul piano tematico che su quello formale, molto spesso metaletterario [...]. Proprio grazie a questa complessità, il libro tende a farsi universo autonomo, a dilatarsi fino a comprendere le voci e le scritture più diverse, a recuperare il rapporto con l'extratestualità a pura funzione della testualità ». Giorgio Patrizi, " Petrolio e la forma del romanzo ", in M.A.Grignani et C. Benedetti, A partire da Petrolio. Pasolini interroga la letteratura, Ravenne, Longo editore, 1998, p. 16-17.

12. "Mi sono caduti per caso gli occhi sulla parola " petrolio " in un articoletto credo dell'Unità, e solo per aver pensato la parola « Petrolio » come il titolo di un libro mi ha spinto poi a pensare alla trama di tale libro. In nemmeno un'ora questa " traccia " era pensata e scritta ». Pier Paolo Pasolini, Petrolio, op. cit., p. 543. 
contemporaine et l'enjeu politique et économique du pouvoir, qui a tout intérêt à contrôler la culture et les rapports de classe. Derrière la longue élaboration de l'ouvrage, l'interrogation presque obsessionnelle sur la notion de forme, et l'ambition de mettre en œuvre un projet global qui sache mêler styles, voix et genres différents, il y a sans doute une lecture stylistique, rhétorique et métalinguistique de Gadda et de son idée de pastiche. La forme du roman, devenant le lieu de l'inachevé, ne peut se composer que de notes, de fragments, d'une structure malléable capable d'accueillir la totalité et le particulier, l'éternité et l'histoire, la passion et l'idéologie, le mythe et le projet dont l'ouvrage se compose. Fondée sur l'opposition et le dédoublement, la morphologie du texte met à profit l'ironie pour pénétrer la complexité du réel, partagé entre sa nature angélique et démoniaque, comme Lukacs l'avait écrit dans sa Théorie du roman. L'ironie devient ainsi la seule objectivité possible du roman ${ }^{13}$.

L'ordre chaotique et le caractère fragmentaire de l'ouvrage reflètent visiblement le désordre de la société italienne, coincée entre le rêve d'une richesse promise par la classe dirigeante et une crise de la dialectique sociale, dont les intellectuels ignoraient - ou faisaient semblant d'ignorer - les conséquences désastreuses. En tout cas la référence au pétrole annonçait déjà l'allusion explicite aux intrigues politiques et financières liées à l'ENI, l'établissement national qui gérait la commercialisation du pétrole.

Dans l'article intitulé " Le génocide », Pasolini fait notamment allusion à une série de notes qui occupent la partie centrale de Pétrole et qui s'intitulent Il Merda. Cette section se compose d'une suite de visions représentant la dégradation de la jeunesse italienne, dont un jeune homme de vingt-cinq ans, "le Merde ", est le prototype. En se promenant avec sa fiancée Cinzia dans une rue de la banlieue de Rome, la via Tuscolana, ils deviennent les jouets de cet ensemble de visions qui ont lieu dans les ruelles transversales de la via Tuscolana. Chaque ruelle représente une espèce de fosse ou de cercle infernal, qui renvoie explicitement à la structure dantesque de la Divine comédie. À l'entrée de chaque ruelle, les deux jeunes gens se retrouvent face à un tabernacle représentant l'une des nouvelles valeurs sociales imposées par le pouvoir. Les jeunes se conforment

13. "L'ironia dello scrittore è il misticismo negativo dei tempi che non hanno dei : una docta ignorantia di fronte al senso ; una esibizione dell'agire, benevolo o malevolo, dei demoni ; la rinuncia a comprendere oltre il mero fatto di questo agire e la profonda certezza, esprimibile soltanto coi mezzi della creazione artistica : quella di aver realmente raggiunto, scorto e colto, in questo non voler sapere e non poter sapere, il termine ultimo, la vera sostanza, il dio presente e inesistente. Perciò l'ironia è l'obiettività del romanzo ». G. Lukács, Teoria del romanzo, trad. it., Milan, Sugar, 1972, p. 321. 
rapidement à ces modèles de vie, perdant à jamais leurs anciennes habitudes et leur culture d'origine. Ces visions, proposées par Pasolini à travers un langage et une forme littéraires, symbolisent une véritable descente aux enfers du monde contemporain et expliquent très clairement la position de l'auteur face à ce génocide culturel. Même s'il ne s'impose pas par la force armée, le nouveau pouvoir poursuit son projet, tout en poussant les classes subalternes à avoir honte de leur situation sociale, dans le but de les convertir à " la religion de la consommation ». L'hédonisme "interclassiste " proposé par la culture bourgeoise force les nouvelles générations à imiter les comportements d'une classe moyenne qui n'a pas conscience d'être à son tour l'instrument dont le " nouveau pouvoir " se sert pour créer une société déshumanisée.

Outre l'hédonisme " interclassiste ", Pasolini énumère, parmi ces nouvelles valeurs comportementales, la fausse tolérance (qui exclut toute forme de compréhension de la différence), l'abjuration de la tradition, le conformisme, la dignité virile, le laïcisme, et, enfin, la nouvelle criminalité. La nature ambiguë et violente de ces valeurs serait à l'origine d'une sorte d'aphasie : un nivellement linguistique qui s'instaure après la disparition de l'élément créatif de la langue, jadis perceptible dans la différence et la richesse des dialectes et des parlers locaux ${ }^{14}$. Cette corruption du langage - de la langue orale et maternelle - s'exprime par la restauration d'une langue littéraire moyenne dépourvue de toute qualité créative. Dès lors, la langue ne peut qu'informer, que devenir un slogan, un simple acte de communication ${ }^{15}$.

14. «Le modèle que la classe dominante a déposé dans ces lieux les a bloqués linguistiquement. À Rome, par exemple, on ne sait plus inventer, on est tombé dans une sorte de névrose aphasique où l'on parle une fausse langue, qui ne connaît ni difficulté, ni résistances, comme si tout était facile à mettre en mots : on parle comme dans les livres imprimés. Ou alors on en est carrément à la pure et simple aphasie au sens clinique du mot : on est incapable d'inventer des métaphores et des mouvements linguistiques réels, on gémit presque, ou l'on se donne des bourrades, ou bien l'on ricane sans savoir quoi dire ". Ecrits corsaires, op. cit., p. 263-264.

15. Au-delà de la perte des langages et des parlers locaux imposée par les moyens de communication de masse, tels que les quotidiens, la radio, et surtout la télévision, gérés de plus en plus par la classe sociale au pouvoir, on assiste, après les premiers succès littéraires du néoréalisme, à une restauration linguistique qui concerne aussi le domaine littéraire. L'échec linguistique et culturel de l'expérience néoréaliste représente pour Pasolini la faillite des principes de la Résistance, qui n’ont pas su pénétrer la société italienne et ont rapidement épuisé leur puissance révolutionnaire pour faire place à une culture moyenne et conformiste : "Già alla fine degli anni cinquanta si avevano i primi sintomi di una crisi che allora pareva di restaurazione. Quale informazione rara, poco nota ai non addetti ai lavori, collocherei l'inizio di tale crisi nella "reazione puristica " (reazione a quelle ricerche plurilinguistiche, dialettali, sperimentali, che erano state la forma letteraria concreta dell'impegno) dovuta all'iniziativa di un gruppetto di scrittori napoletani riuniti intorno a una loro rivista. Tuttavia, protagonisti, in parte involontari, di simile reazione, consideriamo pure Cassola e Bassani, attraverso la loro disperata e poetica nostalgia borghese. Il loro stile (l'ho accennato) non è che una serie continua e sia pur coperta di « citazioni » del linguaggio borghese e piccolo-borghese usato dai padri e dai nonni professionisti [...]. E' vero anche che il 
Le but de la nouvelle société de consommation, fondée sur le principe de la «standardisation ", étant de faire naître les mêmes besoins chez tout le monde, il fallait par conséquent qu'il y ait une conscience partagée d'appartenir à un même niveau social. La lutte des classes devait ainsi apparaître comme un système dépassé. Mais, pour pousser les classes sociales les plus démunies à consommer, il fallait leur accorder des bénéfices, des concessions : on leur promettait de participer un jour à la richesse, à un progrès généralisé, qui allait leur coûter très cher. Elles achetaient un bienêtre économique partiel au détriment de leur identité culturelle ${ }^{16}$.

Ce "pouvoir obscur ", qui se présente plutôt comme un vide de pouvoir, repose sur la séparation entre conscience et existence. Dans un pays comme l'Italie, qui n'a jamais connu de véritable révolution de gauche, le travailleur (l'ouvrier, le paysan, etc.) vit dans sa conscience l'idéologie marxiste et, par conséquent, l'idée de progrès ; en même temps - et à l'inverse - il vit dans son existence l'idéologie de consommation, et donc les valeurs du développement. De cette dissociation, menant l'homme à la perte de toute maîtrise de sa vie et de son identité, profite ce " pouvoir obscur » : il vise à réaliser une société fondée sur un développement multinational - ou transnational -, effaçant d'un coup d'éponge le fascisme traditionnel, et donc l'idée de nationalisme et de cléricalisme sur lesquels la même société reposait auparavant. Ce système bipolaire de séparation entre conscience et existence, progrès et développement, pouvoir et antipouvoir, conduit l'homme moderne à vivre dans un état permanent de schizophrénie.

Pour revenir à la section Il Merda de Pétrole, on s'aperçoit que Pasolini emploie, sur le plan littéraire, une écriture reproduisant l'effet de la vision

discorso rivissuto in funzione di denuncia di un mondo miserabile, ladro, affamato, disponibile perché preistorico, sembra d'improvviso un fenomeno stilistico superato ", Nuove questioni linguistiche, in Pier Paolo Pasolini, Empirismo eretico, Milan, Garzanti, 1972, p. 12-13.

16. "Voyons : le mot " développement » a aujourd'hui tout un réseau de références qui concerne un contexte indubitablement de "droite ». Qui veut en effet le "développement »? [...] C'est évident : ce sont ceux qui produisent qui veulent ce développement-là : les industriels. [...] La technologie (application de la science) a créé la possibilité d'une industrialisation pratiquement illimitée et dont les caractères sont déjà concrètement transnationaux. Les consommateurs de biens superflus sont, pour leur part, irrationnellement et inconsciemment d'accord pour vouloir ce développement. Pour eux, il signifie promotion sociale et libération, avec pour conséquence l'abjuration des valeurs culturelles qui leur avaient fourni les modèles du "pauvre ", du " travailleur », de l'« épargnant ", du "soldat ", du " croyant ». La masse est donc pour le " développement " : mais elle ne vit cette idéologie qu'existentiellement, et c'est existentiellement qu'elle est porteuse des valeurs nouvelles de la consommation. [...] Qui, par contre, veut le progrès ? Ceux qui n'ont pas d'intérêts immédiats à satisfaire à travers le progrès : les ouvriers, les paysans, les intellectuels de gauche. Le veulent ceux qui travaillent, et qui sont donc exploités. [...] Le progrès est donc une notion idéale (sociale et politique), alors que le développement est un fait pragmatique et économique", in Écrits corsaires, op. cit., p. 226-227. 
et reposant sur la technique du dédoublement. Le lecteur se trouve face à une double scène : celle du réel, et celle, plus onirique, de la vision. La première représente la perte progressive des anciennes valeurs, la seconde fait allusion à un présent qui n’a aucune influence sur l'histoire. À plusieurs reprises, l'auteur parle d'un présent social, culturel et économique qui bascule dans le domaine de l'" après-histoire » : en dehors, donc, du domaine de l'actualité. Il Merda nous révèle le passage de la vie de l'homme d'un cycle naturel - reposant sur la succession harmonieuse des saisons - à un cycle industriel - celui de la société de consommation - qui déclasse l'idée d'humanité. L'homme devient une marchandise dans les mains du pouvoir, presque un déchet du processus de production et de consommation.

Entreprenant son voyage à travers les nouvelles valeurs proposées par notre société capitaliste, il Merda vit une véritable conversion a contrario, si l'on prend pour modèle de conversion celle de saint Paul sur le chemin de Damas. On assiste donc au passage d'un état d'innocence, de pureté, d'intégrité originelle, à un état de laideur physique et morale, de pauvreté culturelle qui réalise, enfin, le projet hédoniste de la classe et de la culture dominantes. C'est la disparition de toute dialectique entre les différentes classes sociales et, par conséquent, la mort de tout idéal, pour laisser la place à un simple pragmatisme ${ }^{17}$. Plus tard seulement, on s'apercevra que derrière cette idée de développement se cachait l'anéantissement du sentiment de liberté, du caractère spécifique de l'individu : comme les paysans des campagnes, les prolétaires représenteront toujours des hommes " hors du temps " et " hors de l'espace », tout en participant à la stratégie de terreur mise en place par le même pouvoir qui, pour garder son contrôle sur les masses et pour les désorienter, n'avait pas d'autre possibilité que de recourir à la violence et au désordre social.

17. "L'estraneità dalla classe dei ricchi è totalmente scomparsa dai loro corpi. La pasta di tali corpi, che era appunto l'estraneità dalla storia ufficiale a impastare, è rimasta è vero una pasta povera, che spira scarsezza e alterità. Un nuovo destino, però, insieme a quello antico - ormai senza forze - si è dato da fare a impastare quella carne. Anziché massacrarla e cancellarla dalla faccia della terra in un genocidio cruento, ha trovato una soluzione finale molto più facile : rimpastarla. Eccola. Pallida e livida, con l'occhio spento, vuoto. La furberia come un pianto rappreso ; il ghigno come un'implorazione, moncata sul nascere, di pietà. [...] Il loro ideale di vita è rappresentato dai professionisti o dagli impiegati che derubano o rapinano. Il vuoto lasciato dalla vita che si è ritirata dal loro corpo come un'acqua che prosciugandosi lascia la riva piena di fetenti immondizie, è riempito dalla dignità borghese, professionistica, tecnica, organizzativa, la cui Volgarità, unita alla Miseria che ancora ad ogni modo persiste in quei Corpi, spira il sacro orrore di un corpo seviziato e assassinato ", in Petrolio, op. cit., p. 370-371. 


\section{La violence du pouvoir : désacralisation du corps et stratégie de la tension}

La violence de ce pouvoir est encore évoquée dans le film Salò ou les 120 journées de Sodome. Pasolini situe les sarabandes infernales et les pratiques sexuelles délirantes décrites par le marquis de Sade dans l'atmosphère contraignante et maladive de la République de Salò. Il fait de cette institution une métaphore cruelle de l'exercice d'un pouvoir arbitraire et insensé. Par la pratique de cérémonials, le respect obsessionnel de la forme, la coercition absolue, le fascisme de Salò évoque visiblement une sorte de fascisme contemporain qui, aux yeux de Pasolini, est encore plus dangereux que la dictature de Mussolini. C'est la métaphore d'un pouvoir qui, tout en respectant la démocratie et tout en jouant sur la passivité d'une opposition presque absente, dénie tout principe de liberté en humiliant l'homme jusqu'à le priver de son corps. Derrière l'image du développement et de la richesse économique, ce type de pouvoir parvient à cacher son véritable projet : la disparition de toute culture, de toute identité sociale au nom d'une seule vérité et de l'affirmation des nouvelles valeurs de la bourgeoisie moyenne ${ }^{18}$.

Dans cette perspective, le film propose un univers clos et sans issue, une suite de rituels vides, tels que de faux mariages, des repas hors du commun, des inspections cruelles : bref, un "Lager " parfaitement organisé sur le principe inéluctable d'une obéissance ne permettant aucune possibilité de révolte générale. Pourtant, tout au long du film, des actes d'opposition sont évoqués; il s'agit de révoltes individuelles, donc inutiles, comme dans la séquence montrant un jeune homme qui salue le poing fermé, à la manière des communistes, après avoir été surpris lors d'un acte sexuel avec

18. Dans son dernier film, Salò ou les 120 journées de Sodome, sorti à Paris après sa mort, en novembre 1975, Pasolini semble résumer, à travers les trois cercles dont le film se compose, les différentes phases du parcours de ce pouvoir atteint de cécité. Il illustre, tout d'abord, la perversion du pouvoir à travers les obsessions sexuelles des quatre bourreaux-protagonistes. Après cette première phase, le film montre le cercle de la merde, symbole d'un corps-marchandise considéré par le pouvoir en tant que résidu inorganique du cycle industriel de production et consommation de la société post-moderne : "Se nei film della Trilogia, non a caso ispirati da opere scritte in secoli passati, l'esercizio del sesso è un momento liberatorio e di irrisione al potere, in Salò si riduce a mercificazione e sfruttamento, e può essere esercitato solo per costrizione o di nascosto. Il primo impegno dei quattro mostri protagonisti del film, infelici rappresentanti di uno Stato senza popolo che sopravvive alla propria fine (la Repubblica di Salò) è ridurre a cose delle vittime umili, è fare mercato e consumo dei loro corpi e delle loro manifestazioni di vitalità ", in Piero Spila, Pier Paolo Pasolini, Rome, Gremese Editore, 1999, p. 117. Une fois que le pouvoir a agi et a bien profité des avantages qu'il tire des corps des victimes, il n'a plus qu’à s'en débarrasser. Et voilà, enfin, le cercle du sang, où toutes les formes de tortures, d'amputations et de tueries rituelles sont admises. Le projet de ce " pouvoir obscur " s'achève donc par la disparition physique et culturelle des classes populaires, les véritables victimes qui ont permis à ce même pouvoir de réaliser ce que Pasolini appelle "génocide culturel ". 
la serveuse noire de la villa; ou encore, la scène du suicide de la pianiste qui préfere la mort en évitant ainsi d'être témoin des tortures cruelles perpétrées par les quatre notables.

Tourné dans une atmosphère de cruauté presque insoutenable, ce film représente le voyage de Pasolini dans la nuit de notre époque contemporaine, dans l'horreur d'une société "standardisée " par un non-pouvoir et désormais peuplée de robots qui ont du mal à exprimer leurs sentiments, leur caractère spécifique ${ }^{19}$.

L'analyse de Pasolini concernant la violence de ce type de pouvoir, qui dépasse largement le domaine politique et les événements historiques de l'époque (les mouvements de 68, la polémique avec les étudiants, les réformes sociales liées à la loi sur l'avortement et sur le divorce), se poursuit dans un article bouleversant qui remet en question le rôle des intellectuels et leurs responsabilités face à la société. Intitulé « Le roman des massacres », ce texte exprime l'attention et l'amour que Pasolini portait à son pays, rattachant la notion de responsabilité civique aux domaines littéraire et culturel. L'écriture devient ainsi une forme claire de témoignage, d'où résulte la relation entre littérature et histoire, entre histoire et actualité ${ }^{20}$.

19. «Pasolini gira e monta Salò con un rigore e una durezza che sgomentano (scoppiano anche delle polemiche per i metodi di lavoro che sarebbero stati imposti sul set) ; lo fa quasi a sottolineare la scelta personale di vivere dentro l'inferno che sta mettendo in scena, nonché la sfida di rappresentare il cuore della violenza fino all'estremo limite consentito. E forse oltre. A chi gli rimprovera i suoi metodi, Pasolini risponde giustificandosi con le ragioni del lavoro. "Se uno dei miei attori deve cadere a terra morto scrive in una pagina del diario di lavorazione - glielo faccio ripetere mille volte finché sembra proprio un corpo che cade morto" ". In Piero Spila, Pier Paolo Pasolini, op. cit., p. 119.

20. Le Roman des massacres a été écrit le 14 novembre 1974 et il a été publié dans le Corriere della sera sous le titre "Qu'est-ce que ce golpe?" (Écrits corsaires, op. cit., p. 131-137). Dans cet article, Pasolini affirme connaître les noms des responsables de différents massacres qui ont eu lieu en Italie de 1969 à 1975. Le 12 décembre 1969, une bombe explose à Milan, Piazza Fontana, faisant seize morts et une centaine de blessés. Le même jour, deux attentats à la bombe se produisent à Rome, où une vingtaine de personnes seront gravement blessées. Les autorités accusent d'abord des groupes d'anarchistes ; les enquêtes judiciaires mènent surtout à l'accusation Pietro Valpreda qui, après trois ans de prison, sera acquitté en 1985, et de Giuseppe Pinelli. Ce dernier, après avoir passé deux nuits en prison, se suicide en se jetant par la fenêtre du bureau du commissaire Calabresi. La piste des anarchistes devenant de plus en plus douteuse et incertaine, le tribunal de Milan change d'hypothèse et ouvre une enquête sur des groupes néofascistes de la région de Venise rattachés à Franco Freda et Giovanni Ventura, qui était proche de Guido Giannettini, colonel des Services secrets de la défense (SID), et du parti d'extrême droite, le MSI. Un certain nombre d'enquêtes sont diligentées contre les services secrets de l'État, mais la plupart des fichiers du SID demeurent inaccessibles aux juges. Après une condamnation à la prison à perpétuité, Freda et Ventura sont acquittés par la cour d'appel. Le massacre de la Piazza Fontana reste à nos jours sans responsable. Pasolini fait aussi référence à un coup d'État organisé la nuit du 7 au 8 décembre 1970, par Junio Valerio Borghese, commandant en chef à l'époque de la République de Salò. Avec la complicité d'un bataillon de parachutistes sous la direction de Sandro Saccucci, futur député du MSI, il parvient à occuper le ministère de l'Intérieur pendant quelques heures. Les Italiens n’ont été mis au courant de l'événement que trois mois après. En 1974, quatre généraux sont accusés d'avoir été complices de Borghese, mais ils sont tous acquittés. Dans son article, Pasolini parle aussi de l'attentant à la bombe de Brescia, organisé lors d'une manifestation antifasciste provoquant huit morts, et de celui qui eut lieu la même année, en août 1974, dans un train entre Florence et Bologne, où douze 
Pasolini évoque remarquablement la différence qui s'instaure entre l'intellectuel et le politicien. Il estime connaitre les noms des responsables des massacres mais, en même temps, il dit ne pas pouvoir donner de noms car il n'a ni preuves ni d'indices. Le savoir de l'intellectuel et celui du politicien sont très différents : le premier peut avoir un sentiment de vérité car il ne vit pas la réalité à travers les compromis qu'elle requiert pour se faire comprendre ; l'écrivain manque de pragmatisme, de pratique vis-à-vis d'un pouvoir qui ne peut pas être nommé parce qu'il représente la réalité même. L'homme des institutions, au contraire, pourrait sans doute avoir des preuves et, par conséquent, citer des noms précis. Toutefois, comme il a toujours quelque chose à perdre, il hésite. C'est justement pour cela qu'il ne peut que se taire, refoulant dans le silence le cri de vérité qui restera étouffé. D'un côté se trouve le courage intellectuel de la vérité, qui sait et qui est prêt à prononcer les noms dans le vide, du fait d'un manque de preuves; de l'autre, la pratique politique, qui sait mais ne dit rien car toute forme d'aveu provoquerait une perte ${ }^{21}$. Si le monde ne peut se reconnaître que dans un pouvoir insaisissable qui génère ce même type de violence, il faut supposer une fracture entre l'histoire (la succession des événements) et l'actualité (la conscience de la succession des événements). Tout cela provoque un vide réel par manque de témoins : de ceux qui savent pour avoir "vu ", pour avoir " vécu» :

personnes furent tuées. L'auteur évoque en outre la violence qui éclate au cours des premiers mois de 1975, quand de nombreux désordres ont lieu lors de manifestations politiques fascistes et antifascistes, qui provoquent la mort de Giorgio Mantekas, militant du MSI, de Claudio Varalli et de Giannino Zibecchi, membres du mouvement des étudiants communistes. Cette recrudescence de la violence et de la stratégie de la terreur s'explique si l'on tient compte de trois facteurs importants : avant tout, la crise des groupes révolutionnaires qui, lors des élections de 1975, n’avaient eu aucun succès auprès des électeurs ; ensuite, il faut considérer la fracture irrémédiable qui se creusait entre le PCI, les jeunes des grandes villes et les universitaires qui supportaient mal la collaboration politique qui se préparait entre le PCI de Berlinguer et la DC d'Aldo Moro ; enfin, un renforcement sensible en terme de recrutement et de compétence en matière d'organisation des bandes terroristes qui s'opposaient à la faiblesse de l'armée et des forces de l'ordre (cf. Paul Ginsborg, Storia d'Italia dal dopoguerra a oggi, Turin, Einaudi, 1989, p. 511-513). À propos de cette attitude de l'État face aux groupes armés, l'historien G. Galli écrit : "Le forze di sicurezza che, come si è visto, erano sin dall'inizio sempre state in grado di fronteggiare il partito armato, persero apparentemente di colpo questa capacitsà proprio mentre le Brigate Rosse preparavano l'operazione più clamorosa dell'intera storia della lotta armata... ". G. Galli, Storia del partito armato, 1968-1982, Milan, Garzanti, 1986, p. 152.

21. À propos de cette fracture entre homme politique et intellectuel, qui concerne aussi l'opposition entre courage intellectuel et pratique politique, Pasolini écrit : "On pourrait mobjecter que moi, par exemple, en tant qu'intellectuel et inventeur d'histoires, je pourrais entrer dans le monde explicitement politique (du pouvoir ou de ce qui l'entoure), me compromettre avec lui et donc faire partie de ceux qui ont le droit d'avoir, avec pas mal de chance, des preuves et des indices. Mais je répondrai à cette objection que cela n'est pas possible, parce que c'est justement ma répugnance à entrer dans un tel monde politique qui s'identifie avec mon courage intellectuel potentiel pour dire la vérité, autrement dit les noms ". Écrits corsaires, op. cit., p. 134. 
Je sais parce que je suis un intellectuel, un écrivain, qui s'efforce de suivre tout ce qui se passe, de connaître tout ce que l'on écrit à ce propos, d'imaginer tout ce que l'on ne sait pas ou que l'on tait ; qui met en relation des faits même éloignés, qui rassemble les morceaux désorganisés et fragmentaires de toute une situation politique cohérente et qui rétablit la logique là où semblent régner l'arbitraire, la folie et le mystère. Tout cela fait partie de mon métier et de l'instinct de mon métier. (ibid., p. 133)

La corruption de ce pouvoir invisible, n'admettant ni témoins ni preuves, ni noms ni paroles, agit aussi grâce à la complicité d'une opposition politique qui, ne proposant pas d'autres formes possibles, répond à la cruauté de l'ancien pouvoir par un contre-pouvoir ancré aux institutions mêmes qu'il prétend faire disparaître. Pasolini reprochait ainsi au Parti communiste l'inefficacité de sa politique d'opposition fondée sur l'attachement et la proposition de certaines valeurs moralisantes et dépassées qui faisaient référence à la nomenclature et aux schémas dégradants et passéistes de la classe politique au pouvoir : «En conséquence, les hommes politiques de cette opposition ne peuvent manquer de se comporter eux aussi comme des hommes de pouvoir » (ibid., p. 136-137).

\section{La violence du pouvoir ou la liberté de ne pas être libre}

Dans le recueil poétique Trasumanar e organizzar, Pasolini consacre plusieurs poèmes à la polémique contre un Parti communiste qui opérait une distinction entre vérité politique et pratique politique. Cette dualité était un alibi pour permettre à l'opposition de se compromettre avec le pouvoir. Elle était impossible à réaliser pour un intellectuel qui ne ressentait pas l'exigence de se révolter contre un " père " pour exercer et produire ensuite le même système institutionnel présent auparavant. Dans La poesia della tradizione (La poésie de la tradition), qui fait partie de la section intitulée Poemi zoppicanti (Poèmes boiteux), Pasolini, se référant aux récentes manifestations des étudiants membres des organisations communistes, écrit :

Oh, generazione sfortunata, / arriverai alla mezza età e poi alla vecchiaia / senza aver goduto ciò che avevi diritto di godere / e che non si gode senza ansia e umiltà / e così capirai di aver servito il mondo / contro cui con zelo " portasti avanti la lotta " : / era esso che voleva gettar discredito sopra la storia - la sua ; / era esso che voleva far piazza pulita del passato - il suo ; / oh, generazione sfortunata, e tu obbedisti disobbedendo ! / Era quel mondo a chiedere ai suoi nuovi figli di aiutarlo / a contraddirsi, per continuare.

La génération des jeunes communistes italiens, comme les syndicats ouvriers et les associations contestataires, se seraient battus, plus ou moins consciemment, pour en arriver en fin de compte à adapter les anciennes institutions au nouveau système de consommation, réalisant une société 
qui a perdu la mémoire de ses traditions et de son histoire. Autrement dit, pour Pasolini, c'est le triomphe d'une sous-culture, où tous peuvent se sentir des " habitants parfaits" d'une même maison dont les fondations reposeraient sur les différentes phases de réaction et de révolte que les Italiens ont vécu pendant les années 60 et $70^{22}$.

En Italie, la culture de gauche a tenté pendant toute une décennie de combler un vide par l'emploi de la raison et le refoulement de l'histoire. Aucune révision de l'Etat n'a eu lieu : on a assisté à la disparition d'une époque " clérico-bourgeoise » et à l'adaptation généralisée à une société de consommation qui a fait de la lutte politique un alibi, pour faire croire à l'opposition qu'une possibilité de changement était encore envisageable. En réalité, cette violence politique et sociale n'a créé que des illusions : un rêve de liberté, d'une égalité qui se transforme en «standardisation » culturelle, d'une émancipation camouflant les limites d'un développement sans aucun progrès, dont le but était d'uniformiser, pour que chacun ait les mêmes nécessités. De vide en vide, pour que tout reste immobile et surtout gérable, avec ou sans violence.

\section{La violence du pouvoir : le langage littéraire au-delà d'une forme}

À la lecture des différents passages des Écrits corsaires, on s'aperçoit que la
défaite de Pasolini relève surtout de son impossibilité à pouvoir "dire», en
raison d'une « aphasie " qui ne peut trouver une dernière issue que sur le
terrain littéraire $^{23}$ : il peut atteindre à la vérité par une sensibilité évoca-

22. Le recueil poétique Trasumanar e organizzar, qui comprend les poèmes écrits par Pasolini entre 1968 et 1970, présente une section qui s'intitule La restaurazione di sinistra (La restauration de la gauche), consacrée à la polémique entre Pasolini et l'action et le comportement de tous ceux qui se reconnaissent en tant que militants de gauche. Au lieu de combattre l'ancien pouvoir par la négation de toute notion d'ordre, ils semblent finalement avoir participé, très activement, à la constitution d'un ensemble social portant sur des principes normatifs bien précis. Ils n'ont pas changé les règles et les points de référence de la société italienne. Au contraire, ils ont bâti un système " autre " en renonçant à leur propre diversité, à leur propre identité sociale, culturelle, politique. «I ragazzi manifestarono inquietanti analogie coi padri / almeno su questa faccenda degli allori, della degradazione / e della buona reputazione : / la serietà prima di tutto ". La restaurazione di sinistra, in Trasumanar e organizzar, op. cit., p. 130 ; "Dall'altra parte, ci fu la resurrezione dei Sindacati / che portarono avanti nello spazio vuoto che si era aperto / le grandi masse degli operai con la loro coscienza di classe. / Sicché, per questa operazione, / l'antico prestigio dei Partiti comunisti fu restaurato. / Nei visi dei giovani la presente primavera - / chi non disubbidisce lascia il posto all'obbedienza / che, se perde qualcosa, la perde per sempre / come insegna, inascoltato o non compreso, il tempo nuovo / che si aggiunge al vecchio ". La restaurazione di sinistra (III), ibid., p. 132 ; " Gli studenti di tutto il mondo - / I Partiti Comunisti e i Sindacati stettero a guardare ; / poi venne il loro turno. / Il vuoto fu riempito e adesso padroni e operai / si trovano di fronte più avanti ». La restaurazione di sinistra e chi, ibid., p. 133-134.

23. "E' questa saggistica politica d'emergenza la vera invenzione letteraria degli ultimi anni di Pasolini. Si fonda sullo schema retorico della requisitoria, ed è la grande oratoria di accusa e di autodi 
trice, au-delà de toute description. L'écrivain est forcé de parler de ce pouvoir, de ce vide, à travers le langage même du pouvoir, et - comme Alfonso Berardinelli l'a bien montré dans sa préface à l'édition italienne des Écrits corsaires - Pasolini entame son discours sur le nouveau pouvoir, pragmatique et écrasant, en usant d'un langage uniforme et riche en argumentations, comme s'il se situait dans un processus mimétique. D'ailleurs, la valeur de ces écrits pasoliniens ne réside pas dans l'originalité des contenus : au contraire, la force vient surtout de son raisonnement honnête et " géométrique ", exprimant toute son angoisse pour la perte irrémédiable du caractère sacré de la réalité. Il se sent face à un temps qui manque de "trasumanar", au profit d'un abandon total et sans réserves à un organiz$z a r$ fondé sur le vide des certitudes et sur les idées reçues de la majorité. Dans Écrits corsaires, Pasolini fait preuve d'un engagement civil qui passe par une idée différente du langage et de la littérature. Face à la "standardisation » des traditions, des cultures, des passions et des besoins, de la langue et des comportements, il essaye, comme dans toute sa dernière production, de refonder une "forme » littéraire proposant au lecteur un texte ouvert, une fabula infinie, où toute forme de langage peut retrouver son expression spécifique, le caractère sacré du réel. Il ne s'agit pas de textes à lire, mais d'hypertextes à vivre, à compléter, à composer, à décomposer, à reconstituer. On ressent, chez l'auteur, le besoin de créer une action dans le langage, un mouvement de recherche qui suive deux directions : l'une, de l'auteur vers le lecteur, l'autre, du lecteur vers un autre lecteur, tout en mettant en œuvre un véritable réseau de pensée qui s'appuie constamment sur la rigueur de l'idéologie et l'urgence de la passion ${ }^{24}$.

Pasolini était conscient que la réalité ne pouvait être figée dans une forme : la réalité étant devenue insaisissable (du fait d'une raison corrompue et aveugle), il était désormais impossible de la représenter par des

fesa pubblica di un poeta. Gli stessi toni dell'ideologia sono qui trascinati dalla semplicità contundente dell'argomentazione. Quella degli Scritti Corsari è un'ideologia "vocale ", a braccio, che si muove sull'improvvisazione polemica e su una nitida architettura di concetti, di nervature razionali nude, che sostengono il fragile edificio del discorso con la forza dell' iterazione. Sparisce ogni gioco di sfumature, di attenuazioni, correzioni, incisi, luci e ombre. In questi nuovi poemetti civili o incivili in prosa tutto è disperatamente e rigoristicamente in piena luce " (préface d'Alfonso Berardinelli au volume Scritti corsari, op. cit., p. XII).

24. Cette idée de créer une nouvelle "forme " littéraire ouverte, que l'on pourrait définir en tant qu'hypertexte, est exprimée dans la note introductive à Écrits corsaires, écrite par l'auteur même, où l'on lit : "C'est au lecteur de reconstruire ce livre ! À lui de réunir les fragments d'une œuvre dispersée et incomplète, à lui de faire se rejoindre des passages placés loin les uns des autres et qui pourtant se complètent ! À lui d'organiser les moments contradictoires en recherchant leur unité essentielle, à lui d'éliminer les éventuelles incohérences (hypothèses abandonnées ou de recherche) ! À lui de substituer aux répétitions leurs variantes éventuelles (ou alors d'accepter ces répétitions comme des anaphores passionnées) ". Écrits corsaires, op. cit., p. 23. 
formes et un langage littéraire traditionnels. En ce qui concerne ce recueil d'écrits journalistiques, on a évoqué la tentative de proposer des textes aux limites du littéraire, sur lesquels le lecteur doit opérer une véritable reconstruction philologique pour comprendre une réalité dépourvue d'actualité. Mais à propos du langage, on relève une faille par rapport aux intentions de Pasolini de représenter la réalité italienne des années 1970 - faille dont Jean-Michel Gardair a évoqué l'importance lors d'un séminaire organisé à Catane à l'occasion du vingt-cinquième anniversaire de la mort du poète. Elle concerne l'emploi excessivement mimétique de la langue du pouvoir, dont Pasolini essaie de montrer la violence et l'hypocrisie ${ }^{25}$. Si, d'un côté, on reconnaît à l'auteur le mérite d'avoir créé une écriture politique née de l'urgence d'exprimer son amour pour un pays ébranlé par un pouvoir violent et amorphe, de l'autre, on lui reproche une absence de sens critique et d'esprit dialectique, résultant d'un langage et d'une manière d'argumenter fortement mimétiques par rapport à un pouvoir qui exprimait de la même manière sa fausse tolérance et ses faux interdits.

Tout en s'opposant au projet de Pasolini de créer un ensemble de textes s'adressant à l'intelligence de chaque lecteur (ouvrant ainsi un "dialogue possible » avec l'autre), cette langue mimétique permet toutefois, avec une profonde lucidité d'analyse, de mener un diagnostic, parfois précis, de la situation italienne de l'époque face à un pouvoir qui écrase l'individu et l'idée même d'existence :

La consommation consiste, en effet, en un pur et simple cataclysme qui, du moins pour l'instant, n'est que dégradation : je le vis chaque jour, dans les formes de mon existence, dans mon corps [...]. C'est de cette expérience, existentielle, directe, concrète, dramatique, corporelle, que naissent en conclusion tous mes discours idéologiques. Parce qu'elle est transformation (pour l'instant dégradation) anthropologique des gens, la consommation est pour moi une tragédie, qui se manifeste par une désillusion, une colère, un taedium vitae, une paresse et, enfin, une révolte idéaliste, un refus du status quo ${ }^{26}$.

25. " Ma se si deve finalmente auspicare e tentare una lettura "poetica " del Pasolini luterano e corsaro, non si può non dissentire da chi vede in questa saggistica politica d'emergenza la vera invenzione letteraria degli ultimi anni di Pasolini. L’orgia critica (del Pasolini corsaro e luterano) che sconvolge i codici e le regole di ogni civile dialettica politica, tra fascismo e antifascismo, fino a minacciare, attraverso le forzature (pirandelliane) di un dialogo sregolato, l'affidabilità logica del linguaggio stesso, non è che il sintomo, ossia la forma infelice (terrorista e totalitaria) di una miseria sessuale che solo in Orgia e Petrolio trova la felicità espressiva (sia pure incompiuta) della sua sublimazione poetica (ascetico-cosmica), fuori dai rimpianti esistenziali e storico-epocali ». Jean-Michel Gardair, "L'orgia critica. Tra Marx e Sade ", in Giuseppe Savoca (édit.), Contributi per Pasolini, Florence, Olschki, 2002, p. 56.

26. Pier Paolo Pasolini, Sacer, in Écrits corsaires, op. cit., p. 155. Cet article a été écrit le 30 janvier 1975 et a été publié dans le Corriere della sera, sous le titre "Pasolini réplique sur l'avortement ». Il s'agit d'une réponse à Alberto Moravia qui, auparavant, avait traité Pasolini de catholique. À cette époque, en Italie, on débattait au sujet de la loi sur l'avortement. La position de Pasolini étant contre la légalisation sur l'avortement, elle avait surpris l'opinion publique. Il voyait dans cette loi la énième réalisation de la fausse tolérance de la part du nouveau pouvoir qui, tout en affichant la valeur d'une certaine liberté sexuelle vis-à-vis de la majorité des Italiens, ne faisait de la sexualité qu'une convention, une obligation, 


\section{La violence du pouvoir : le langage poétique comme forme de témoignage}

En 1968-1969, dès la formation et les premières actions des mouvements de contestation, Pasolini avait prévu qu'une vague de violence se préparait ; le signe avant-coureur de ce désordre a été le massacre de la piazza Fontana à Milan. Dans Trasumanar e organizzar, le poète présente un texte, Patmos ${ }^{27}$, évoquant cet événement qui avait bouleversé le pays. Patmos est placé dans la deuxième partie du recueil, parmi les « poèmes politiques »; la parole du poète alterne avec les versets de l'Apocalypse de saint Jean, dans une sorte de contre-chant entre un événement qui fait désormais partie de l'histoire et une vérité qui est pour l'instant annoncée. Ce texte énumère les victimes du massacre de la piazza Fontana : une liste de noms et de prénoms qui forment une sorte de litanie sacrée. Il s'agit de personnes arrachées d'une Histoire qui, par manque de témoins, n'est plus perçue comme actuelle. Cette réalité devient de plus en plus ineffable, à cause de la perte totale du sacré et du sens du mystère, qui s'effacent au profit d'un développement ne pouvant se réaliser que par l'affirmation des nouvelles valeurs de consommation. On ne considère plus l'homme en tant qu'individu : les noms propres qui désignent les victimes se succèdent au long du poème de manière tout à fait anonyme et dépersonnalisante. La répétition des noms évoqués crée une sorte de silence, car cette histoire - que Pasolini, par manque de témoins, définit comme une " après-histoire " - n'est qu'un simple désert. Qu'il soit victime ou bourreau, l'homme n'est plus reconnaissable en tant qu'individu et, par conséquent, le nom propre, perdant à jamais sa fonction d'identification, laisse place à une connotation collective et parfois anonyme. La dimension publique affiche ainsi toute sa suprématie par rapport à la dimension privée, refoulée dans l'oubli par une parole corrompue. Avec un langage poétique proche des nuances tonales de la prophétie, Pasolini, au lendemain du massacre de Milan, écrit une nouvelle Apocalypse de l'époque contemporaine, selon la

un devoir social, une anxiété, une caractéristique inévitable de la qualité de vie du consommateur. À plusieurs reprises, Pasolini a dit que la légalisation sur l'avortement ne représentait qu'une énorme commodité pour la majorité qui s'emparait finalement de certains problèmes de conscience par rapport au coït. À ce sujet, voir. « Le coït, l'avortement, la fausse tolérance du pouvoir, le conformisme des progressistes ", in Ecrits corsaires, op. cit., p. 143-151.

27. Trasumanar e organizzar, op. cit., p. 111-119. Dans un article intitulé "Pasolini recensisce Pasolini », publié dans Il Giorno du 3 juin 1971, le poète explique que dans Trasumanar e organizzar présente toujours la réalité à travers son caractère dramatique, dans une vision non dialectique des événements. L'opposition qu'il relève n'arrive jamais à se résumer dans un moment de pure synthèse ; les éléments opposés continuent à cohabiter tout en s'exprimant par un langage qui procède par oxymores. 
technique du collage. Sur un texte biblique, peut-être parmi les plus difficiles à interpréter, le poète exprime sa vision de l'avenir de la société italienne, tout en évoquant la disparition des traditions paysannes, de la culture ouvrière et donc des racines d'un peuple. Pour Rinaldo Rinaldi, on peut considérer l'auteur comme un nouveau saint Jean, qui rédige l'Apocalypse de la tradition, des valeurs du vieux monde, d'un passé récent qu'on oublie rapidement ${ }^{28}$.

À la disparition de l'histoire du monde va s'ajouter celle de chaque individu et de l'écriture littéraire qui essaie en vain de ramener à la mémoire l'événement destructeur. Le poète ne peut donc que s'éloigner de son écriture, de ses vers qui révèlent au lecteur une sorte de compulsion de répétition. Les martyrs de cette horrible " après-histoire " sont, ainsi, privés même de leur mort, car on est face à un bourreau que l'on ne peut nommer et à des victimes qui ne sont pas reconnues en tant que telles, faute de témoins.

Par ce langage poétique hétérodoxe, fondé sur la répétition presque obsédante du nom propre vidé de toute fonction, Pasolini exprime les origines de cette violence et des massacres qui non seulement sont perpétrés au détriment de l'individu, mais qui visent aussi à faire peur à la masse, cible privilégiée d'une société évoluée et en même temps sous-développée. La violence remplace donc la raison déclassée par un pragmatisme et un langage limité dans sa fonction communicative, au-delà de toute possibilité d'expression. Dans l'irresponsabilité de la violence se résume l'essence du renouvellement de la société et, dans son anonymat, s'éteignent les voix des martyrs qui restent sans corps, sans parole, morts face aux vivants, vivants face à une mort devenue vaine. Toute institution, même si elle naît au sein de la contestation, porte en germe une orthodoxie qui, se développant au fil du temps, demande de respecter un rituel, celui du culte d'un pouvoir se fondant sur un système de référence, autour d'un centre, de la parole des « Pères".

À travers une poésie qui ne veut pas se définir comme engagée ou militante, qui ne veut pas constituer une "forme " reconnaissable, Pasolini fonde pourtant un nouveau modèle de poète : le " poète bouffon ", qui

28. «La struttura segue un rigoroso principio di alternanza. E così all'Apocalisse si intreccia una serie fatta di notizie di cronaca, quindi un commento vagamente nostalgico sugli antichi costumi piccoloborghesi e contadini, infine una sequenza di battute polemiche contro lo Stato e il potere democristiano. L'apocalisse annunciata qui, quella di piazza Fontana, è dunque l'apocalisse della tradizione, del vecchio mondo, del passato. Ma il poeta, questo nuovo profetico Giovanni, è lontano nella sua isola ; la sua parola giunge da un luogo estraneo al presente, alle piccole apocalissi del mondo, e in essa risuona profondamente una distruzione e una rinunzia più grande, quella della storia tout court ». Rinaldo Rinaldi, Lirriconoscibile Pasolini, Rovito, Marra editore, 1990, p. 185. 
transforme et nuance l'urgence de son écriture viscérale par la force de l'ironie. L'auteur lui-même affirme que ce nouveau type de poésie vit dans une zone de la réalité où la réalité est en train de se perdre ou de se dissoudre. Le poète n'est plus indispensable à l'humanité ; c'est pourquoi Pasolini se définit comme un poète amateur, comme un poète dilettante ${ }^{29}$. Il remplace la poésie engagée, fondée sur l'argumentation rationnelle et idéologique, par un langage qui s'appuie sur le parler, la fonction phatique de la parole (laquelle est au-delà de tout code poétique) pour toucher, enfin, une réalité de moins en moins saisissable. Il crée une poésie sans symboles et sans métaphores, un discours poétique didactique et follement autobiographique, où l'Histoire pourrait à nouveau restaurer l'harmonie entre l'individu et le corps social dont il fait partie.

La poésie et, plus généralement, l'écriture de Pasolini dans les années 70, semblent répondre à l'urgence de "parler » du désordre et de la violence qui s'emparent de la société italienne. Tout en se rendant compte de la crise irréversible de l'expression littéraire et de l'intellectuel engagé (qui n'arrivent plus à interpréter le silence et la violence dont le nouveau pouvoir se sert pour gérer la réalité économique, sociale et culturelle du pays), l'auteur ne veut pas être réduit au silence. Se dissimulant dans un dialogue qui ne tient compte d'aucune convention formelle, sa parole arrive à dégager, par la suspension du sens et le caractère fragmentaire de la perception du réel, un surplus d'expression capable de surmonter l'aphasie imposée par le pouvoir de consommation (qui passe par l'anéantissement de la dialectique entre principes opposés). Cette " pratique de la différence », et l'attitude hétérodoxe par rapport à tout système et à toute convention, sont à la base d'une parole se situant dans les intermundia d'un savoir qui se fait humoristique pour exprimer la désillusion. Cette parole tente désespérément de combler le vide du cosmos, où le monde semble se noyer. Pasolini ne tente pas de corriger l'Histoire, mais, au contraire, de la vivre à travers une " diction » qui fait de l'humour la limite ultime de sa lutte et de sa révolte qui restent encore sans écoute.

29. "Al poeta indispensabile per l'umanità, quale egli [Pasolini] si considerava da giovane, subentra ora l'autodefinizione di poeta dilettante ; ad una funzione consumistica della poesia, l'affermazione caparbia e quasi solenne della inutilità della poesia. Da qui il carattere di contingenza ed eterogeneità dei temi presenti in questa raccolta [...]. Il poeta, mentre da un lato sembra guardare con ironia malcelata la propria giovanile posizione di fronte alla poesia-come-centro-dell'universo, dall'altra annuncia che, sulla medesima, la vita ha preso il sopravvento, e che dunque il poeta può, allo stato attuale delle cose, fornire poesie su ordinazione, né più né meno come ordigni ». Luigi Fontanella, "Trasumanar e organizzar di Pasolini : una rilettura », in Sigma, Guida Editore, anno XIV, n. 2-3, maggio-dicembre 1981. 
Au cours de ses dix dernières années de vie et d'activité culturelle, Pasolini a tenté de représenter, au sein du domaine littéraire, la cruauté et le vide d'une violence qui exprimaient la perte de toute forme d'idéologie ; mais il a aussi tenté de transformer le rôle de l'intellectuel post-moderne, auquel on demandait, avant tout, d'être le témoin d'une histoire qui s'éloignait progressivement de la réalité et, par conséquent, de la notion même d'actualité. Après une dure analyse du " paysage italien, humain et social » des années 60 et 70, du désordre chaotique qui s'emparait de toute différence culturelle - d'où la véritable violence de la société de consommation qui allait se mettre en place -, Pasolini oppose, au vide d'un pouvoir déshumanisant et à une forme de sous-culture moyenne et transnationale, la rigueur et, peut-être, l'innocence anticonformiste d'une parole qui fait de l'idéologie une pure passion, à exprimer dans l'urgence de son existence.

À la vague de conformisme, de violence et de désacralisation de l'homme, provoquée par des hommes-ombres, bourreaux et victimes d'une modernisation reniant la notion d'humanité, Pasolini répond par une littérature-action - souvent profuse - qui sollicite et éveille le sens des responsabilités civiques, tente de rendre à chaque individu son rôle social et surtout sa dignité de citoyen. En dépassant la dimension traditionnelle de la littérature et en ouvrant le " texte " à ses différentes potentialités d'expression (pour aboutir presque à un hypertexte ou à un métatexte), Pasolini, au lieu de créer une nouvelle forme de littérature, pratique une écriture hétérodoxe, qui sollicite le lecteur pour qu'il la complète par son inépuisable expérience quotidienne, ses besoins et ses passions.

Contre l'aphasie de l'époque contemporaine, Pasolini propose à la fois la force de l'argumentation et de la critique sociale (Écrits corsaires, Lettres luthériennes ${ }^{30}$ ou encore Empirismo eretico ${ }^{31}$ ), une nouvelle conception de la littérature romanesque fondée sur la notion du mystère et de l'inachevé, la sacralité et les pulsions de l'homme (Pétrole et Divina mimesis ${ }^{32}$ ), et, enfin, une parole poétique de résistance ${ }^{33}$, comme dans le cas de

30. Pier Paolo Pasolini, Lettere luterane, Turin, Einaudi, 1976.

31. Pier Paolo Pasolini, Empirismo eretico, op. cit. (L'expérience hérétique - Langue et cinéma, trad. fr. par A. Rocchi-Pullberg, Paris, Payot, 1976).

32. Pier Paolo Pasolini, Divina mimesis, Turin, Einaudi, 1975. (Divine mimesis, trad. fr. par D. Sallenave, Paris, Flammarion, 1980).

33. Lors d'une interview menée par Jean-Michel Gardair et publiée dans le Corriere del Ticino du 13 novembre 1971, Pasolini, à propos de sa nouvelle manière de faire de la poésie, dit : "Ultimo elemento di rinnovamento linguistico e rinnovamento mio interiore è un certo atteggiamento umoristico verso la realtà, cosa che fino a questo momento non avevo mai avuto perché nei miei romanzi e nei miei film precedenti c'erano momenti di comicità ma quasi mai di umorismo. C’è questo strano fenomeno della perdita di certe illusioni che non si trasforma in scetticismo [...]. Il fatto di scrivere una poesia è già 
Trasumanar e organizzar. À travers la suspension du sens, le caractère fragmentaire et ironique de son œuvre, et l'attitude presque irrévérencieuse du poète-bouffon, Pasolini arrive à rendre visible le vide et le néant d'une histoire déshumanisée, sans passé, sans présent et totalement subordonnée à un futur insaisissable. Il oppose aux certitudes de la société de consommation une littérature d'investigation au-delà de tout système moral, de toute forme, de tout préjugé, pour saisir ainsi la logique se cachant derrière ce type de pouvoir violent et invisible, toujours en quête de complices à réduire au silence.

Il ne nous reste qu’à réfléchir sur la position parfois ambiguë de Pasolini face aux institutions, aux partis politiques qu'il détestait, et surtout face au Parti communiste qui représentait, à ses yeux, un organisme institutionnel renfermé dans un conservatisme presque totalisant. Les deux derniers écrits des Lettere luterane font ressortir l'image d'un Pasolini aligné et favorable à l'action des jeunes communistes (qu'il avait énormément critiqués lors des mouvements de contestation de 1968, mais qu'il considère par la suite comme les « derniers gardiens " d'Italie et de ses pauvres institutions démocratiques). Dans une communication présentée au congrès du Parti radical de Marco Pannella (et publiée le 4 novembre 1975, deux jours après son assassinat), Pasolini déclare avec une certaine force soutenir la position du Parti communiste ${ }^{34}$. Dans certains passages des Écrits corsaires, on retrouve par ailleurs un Pasolini "anti-corsaire», surtout quand il énonce sa profession de foi à l'égard des principes formels de la démocratie $^{35}$.

Cette confiance de l'auteur dans les institutions, vis-à-vis de la politique et des jeunes communistes des années 1970 est une fois de plus trahie si l'on considère ce que Pasolini écrit le 15 juin 1975, dans l'article Abiura dalla Trilogia della vita, publié le 9 novembre 1975 dans le

credere, è già un'illusione. E benché io non m'illuda più come mi illudevo quando ero ragazzo, per me la poesia è stata un'illusione meravigliosa, con tutto quel che di mitico è nei classici. Adesso non credo più a questo, tuttavia continuo a scrivere e non me ne so spiegare il perché ".

34. "Prima di tutto devo giustificare la presenza della mia persona qui. Non sono qui come radicale. Non sono qui come socialista. Sono qui come marxista che vota per il PCI, e spera molto nella nuova generazione di comunisti ". Intervento al congresso del Partito radicale, in Lettere luterane, op. cit., p. 185.

35. "Je ne peux pas ne pas prononcer mon accusation faible et idéale contre toute la classe politique italienne. Et je le fais dans la mesure où je crois à la politique, aux principes formels de la démocratie, au parlement et aux partis. Le tout, naturellement, à travers mon optique particulière, qui est celle d'un communiste [...]. Il est certain qu'aujourd'hui la présence dans l'opposition d'un grand parti comme ce parti communiste italien représente le salut de l'Italie et de ses pauvres institutions démocratiques. Le parti communiste italien est un pays propre à l'intérieur d'un pays sale, un pays honnête à l'intérieur d'un pays malhonnête, un pays intelligent à l'intérieur d'un pays idiot, un pays cultivé à l'intérieur d'un pays ignorant, un pays humaniste à l'intérieur d'un pays consommateur ". Écrits corsaires, op. cit., p. 135-137. 
Corriere della Sera. L'espoir semble céder la place à une résignation irrévocable :

LItalia non sta vivendo altro che un processo di adattamento alla propria degradazione, da cui cerca di liberarsi solo nominalmente [...]. Ma devo ammettere che anche l'essersi accorti o l'aver drammatizzato non preserva affatto dall'adattamento o dall'accettazione. Dunque io mi sto adattando alla degradazione e sto accettando l'inaccettabile. Manovro per risistemare la mia vita. Sto dimenticando com'erano prima le cose. Le amate facce di ieri cominciano a ingiallire. Mi è davanti - pian piano senza più alternative - il presente. Riadatto il mio impegno ad una maggiore leggibilità (Salò ?) ${ }^{36}$.

Au-delà de ses ambiguïtés par rapport au pouvoir, il faut toutefois reconnaître à Pasolini la force humaine et intellectuelle qui lui ont permis de garder une attitude d'innocence face à une société de plus en plus insaisissable. Cette tendance à la démystification du pouvoir et de sa violence se résume dans la courte citation empruntée à Osip Mandel'Stam et choisie comme exergue à Pétrole : "Col mondo del potere non ho avuto che vincoli puerili ${ }^{37}$ ".

\section{Bibliographie}

Seuls les ouvrages critiques et de l'auteur cités et/ou de référence pour la rédaction de cette étude apparaissent dans la liste ci-dessous.

Bazzocchi Marco Antonio, Pier Paolo Pasolini, Milan, Bruno Mondadori, 1998.

Berardinelli Alfonso, Prefazione a Pier Paolo Pasolini, Scritti corsari, Milan, Garzanti, 1990.

Conti Calabrese G., Pasolini e il sacro, Milan, Jaca Book, 1994.

De Ceccatty René, Sur Pier Paolo Pasolini, Le Faouët, Éditions du Scorff, 1998.

De Santi G., Lenti M., Rossini R. (a cura di), Perché Pasolini, Florence, Guaraldi, 1978.

Fontanella Luigi, Trasumanar e organizzar di Pasolini : una rilettura, "Sigma », Guida editore, anno XIV, n. 2-3, maggio-dicembre 1981.

Fortini Franco, Attraverso Pasolini, Turin, Einaudi, 1993.

Galli G., Storia del partito armato 1968-1982, Milan, Garzanti, 1986. Gardair Jean-Michel, L'orgia critica. Tra Marx e Sade, in Savoca Giuseppe (a cura di), Contributi per Pasolini, Florence, Olschki, 2002.

36. Abiura dalla Trilogia della vita, in Lettere luterane, op. cit., p. 75-76.

37. Petrolio, op. cit., p. 7. 
Ginsborg Paul, Storia d'Italia dal dopoguerra a oggi, Turin, Einaudi, 1989. Grignani Maria Antonietta, Benedetti Carla a cura di A partire da Petrolio. Pasolini interroga la letteratura, Ravenne, Longo editore, 1995.

Joubert-Laurencin Hervé, "Pasolini, portrait du poète en cinéaste », Paris, Cahiers du cinéma, 1995.

Lukács G., Teoria del romanzo, (trad. italiana), Milan, Sugar, 1972.

Pasolini Pier Paolo, Divina mimesis, Turin, Einaudi, 1975.

—, Divine mimesis, (trad. fr. par Danièle Sallenave), Paris, Flammarion, 1980.

—, Écrits corsaire, (trad. fr. par Philippe Guilhon), ibid., 1976.

—, Empirismo eretico, Milan, Garzanti, 1972.

-, L'expérience hérétique - Langue et cinéma, (trad. fr. par Anna RocchiPullberg, Paris, Payot, 1976.

—, La nuova gioventù, Turin, Einaudi, 1975.

-, Lettere luterane, ibid., 1976.

—, Pétrole, (trad. fr. par René de Ceccatty), Paris, Gallimard, 1995.

—, Petrolio, Turin, Einaudi, 1992.

—, Scritti corsari, Milan, Garzanti, 1975.

-, Trasumanar e organizzar, ibid., 1971;

Rinaldi Rinaldo, L'irriconoscibile Pasolini, Rovito, Marra editore, 1990.

Spila Piero, Pier Paolo Pasolini, Rome, Gremese editore, 1999. 